\title{
Clustering and decoherence of correlated spins under double quantum dynamics
}

\author{
Claudia M. Sánchez, Rodolfo H. Acosta, Patricia R. Levstein, ${ }^{*}$ Horacio M. Pastawski, and Ana K. Chattah ${ }^{\dagger}$ \\ Facultad de Matemática, Astronomía y Física, Universidad Nacional de Córdoba and Instituto de Física Enrique Gaviola (CONICET), \\ Ciudad Universitaria, X5016LAE, Córdoba, Argentina
}

(Received 30 June 2014; published 30 October 2014)

\begin{abstract}
We present an improved approach for the study of the evolution of spin correlations and decoherence in multiple quantum nuclear magnetic resonance experiments. The infinite system, constituted by the protons of a polycrystalline adamantane sample, evolves under a double quantum Hamiltonian. The distribution of multiple quantum coherence orders is represented by a contribution of spin clusters with different sizes that exchange spins, increasing their size with the evolution time. A cluster with nearly exponential growth at all times is observed, in agreement with previous models. Remarkably, a small cluster that stabilizes in a size corresponding to 18 correlated spins is revealed. In addition, by performing a renormalization of the obtained data with the experimental Loschmidt echo, the contribution of the different clusters to the observable signal is determined. This procedure accounts for the effect of decoherence on the evolution of the system, and allows setting the range of confidence of the experimental data. Our analysis confirms the natural hint that, correlated states involving higher coherence orders are far more sensitive to the uncontrolled decoherent interactions, than those involving lower orders.
\end{abstract}

DOI: 10.1103/PhysRevA.90.042122

PACS number(s): 03.65.Yz, 33.25.+k, 76.60.-k, 75.40.Gb

\section{INTRODUCTION}

Solid-state nuclear magnetic resonance (NMR) represents a unique technique for precise control of nuclear spin dynamics allowing the performance of experimental studies of manybody systems interacting through natural and specifically designed couplings $[1,2]$. The observation of nonequilibrium many-body dynamics $[3,4]$ and the ability to perform quantum time-reversal experiments [5-8]. while assessing the loss of coherence $[9,10]$, have attracted much attention in the last decades, as these issues have an impact on the strategies to handle quantum information.

Decoherence is responsible for the degradation of the information contained in a quantum state. The revival of the initial state after a time-reversal procedure that involves a change in the sign of the Hamiltonian [11-13], i.e., the Loschmidt echo [14], is directly affected by decoherence phenomena. This could be the result of experimental imperfections, the interaction with an uncontrolled environment, and natural instabilities associated with the intrinsic chaos of a system with infinite degrees - as, for example, spins at room temperature $[15,16]$. Indeed, the rate of decoherence increases with the size of the quantum system, as larger systems are more susceptible to perturbations $[7,9]$. The Loschmidt echo (LE) has become the preferred quantifier of decoherence in the context of quantum chaos and quantum information theories $[17,18]$. In NMR, the experimental LE has been evaluated locally in a collection of individual spins acting as "spies" of the spin dynamics $[8,19]$, and globally, by using the total magnetization [20,21]. This information points to distinguishing the decoherence induced by the intrinsic complexity of the system, from the one arising from experimental imperfections.

\footnotetext{
*Deceased.

${ }^{\dagger}$ Corresponding author: chattah@famaf.unc.edu.ar
}

A more detailed study of the multispin quantum dynamics involves not only the analysis of the polarization signal, but also points to the evolution of the different collective manybody states. For this purpose, the experimental generation, detection, and analysis of quantum correlations has been implemented, leading to the distribution of the intensities that sum up those elements of the density matrix that involve quantum transitions of a given order, i.e., the multiple quantum coherences (MQCs) [20,22-24]. The most important information that can be extracted from the MQC distribution is the number of correlated spins, usually known as spin counting, which depends on the evolution time [24]. In recent years, the spin counting and the study of decoherence has been studied for a variety of topologies of the coupling network, for example in liquid crystals and organic crystals, and for different Hamiltonians, where the dipolar and the double quantum have been the most applied [21,25-28].

The most accepted model to extract the number of correlated spins was proposed by Baum et al. [4,24]. The basic reasoning relies on the product operator representation for the evolution of the density matrix [29] popularized by the Ernst group [2]. In this picture, given an initially local excitation [i.e., the density matrix $\rho(0) \propto I_{0}^{z}$ ] the evolution in an elementary time step $\delta t$ can be seen as the superposition of the local excitation and the commutator between $\rho(0)$ and the evolution Hamiltonian. This elementary evolution only contains sites interacting with the local excitation $I_{0}^{z}$. Further evolution steps $(2 \delta t, 3 \delta t$, etc.) increase the number of spin operators connected to the initial excited spin. In consequence, the initially localized excitation spreads out not only as a polarization but also as excitation with different spin projections, which generate multiple quantum coherences (MQCs). The key assumption is, then, that the combinations of spin operators that are summed up in the final states can be considered as having a probability that only depends on the number of pathways that led to them (much as in the classical Galton board [30]). This lack of specific interferences 
is justified by the proliferation of pathways connecting an initial excitation with the possible formation of spin clusters. Thus, the amplitude of each order in the coherence spectrum is provided by counting the number of elements in the density matrix with a certain coherence order, which is given by a binomial distribution. This distribution is approximated, for large numbers, by a Gaussian function in the coherence order.

In our experience, this model describes quite accurately the growth of the clusters in a closed system of few spins in a molecule. An example is a liquid crystal in the nematic phase, where only intramolecular interactions survive with a definite value, leading to a finite proton system [20]. However, as pointed out by Alvarez et al. [31], the Gaussian distribution does not fit well the experimental results in large spin systems. In particular, we have noticed that a single Gaussian fails to describe the distribution of coherences in polycrystalline adamantane at long evolution times, when higher orders of coherence, which account for large clusters of interacting spins, are developed. Although a two-Gaussian model has been proposed in Ref. [24] to describe systems with different spin dilution, its application to homogeneous networks has not received much attention until now.

In this work, we model the system of correlated spins evolving under the double quantum Hamiltonian as composed by an ensemble of clusters that can change their sizes as a function of the evolution time. The protons in polycrystalline adamantane represent our multispin system. The experimental multiple quantum coherence distribution at a given time is assumed to be a distribution of Gaussian functions, where the size of each cluster and the fraction of spins involved in the cluster can be extracted from the width and the area of the associated Gaussian functions, respectively. The experimental strategy adopted here is to renormalize the data with the Loschmidt echo. This procedure compensates the highly attenuated free induction decay signal, recovering the relative relevance of different MQC orders and giving an insight about the effect of decoherence on the evolved system.

\section{MULTIPLE QUANTUM EVOLUTION}

In a strong magnetic field $B_{0}$ in the $z$ direction, a system of $N$ spins $1 / 2$ has $2^{N}$ stationary states. These states can be classified according to total spin projection $M_{z}=\sum_{i} m_{z i}$, where $m_{z i}= \pm 1 / 2$ is the eigenvalue of the $i$ th spin in the system. For nondegenerated stationary states there are $2^{2 N-1}$ possible transitions of finite energy between any two levels. The difference in $M_{z}$ between two coupled states $s$ and $r$ is defined as the coherence number $n$, i.e., $n=M_{z}(r)-M_{z}(s)$ [32].

The density matrix for a system of spins initially in equilibrium with the external magnetic field, expressed in the eigenbasis of the Zeeman system, is $\rho(0) \propto I^{z}$, where $I^{\alpha}$ with $\alpha=x, y, z$ represent the components of the total angular momentum. Here, we have neglected the identity term in the density matrix expansion, as it is invariant under any kind of rotation. The density matrix element $\langle r|\rho| s\rangle$ of an arbitrary state indicates the presence of an $n$ quantum coherence. Note that a rotation around the axis of quantization $\hat{z}$ with an angle $\varphi$ can be used to encode the order of coherence: $\left\langle r\left|\exp \left(-i \varphi I^{z}\right) \rho \exp \left(i \varphi I^{z}\right)\right| s\right\rangle=\exp (i \varphi n)\langle r|\rho| s\rangle[3,4]$.

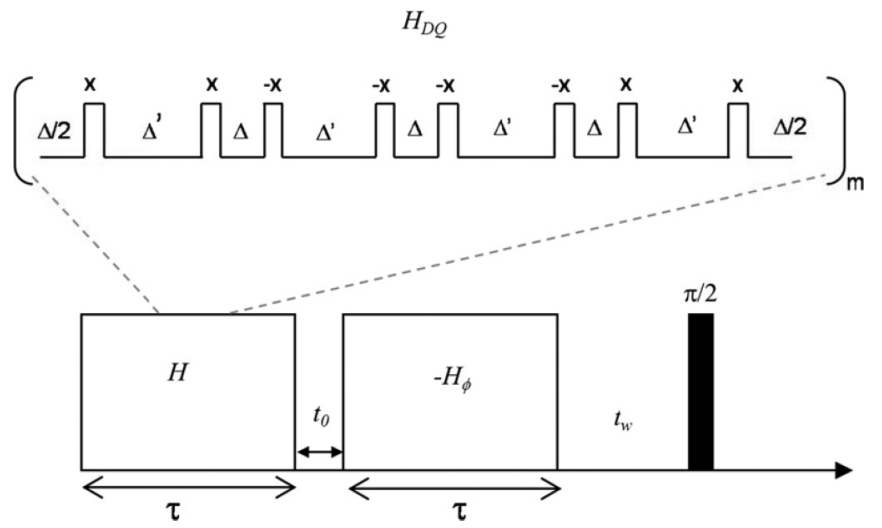

FIG. 1. Schematic representation of the pulse sequence used to study the multiple quantum dynamics under a double quantum Hamiltonian. The reversed Hamiltonian $-H$ is obtained by changing the phases $x$ by $y$ and $-x$ by $-y$. The read-out pulse is cycled with $( \pm y)$.

In this work, the pulse sequence for MQC encoding follows a scheme of excitation, reversion, and detection periods [32]. Figure 1 displays schematically the pulse sequence used in this work. During the excitation period, $\tau$, the system evolves under an effective Hamiltonian $H$, followed by a reversion period $\tau$ under the effective Hamiltonian $-H_{\varphi}$. This Hamiltonian is generated by performing a phase shift, and a rotation around $\hat{z}$ with an angle $\varphi$, to encode the different coherence orders.

The propagator after the total elapsed time for excitation and reversion $\tau+\tau$ is

$$
\begin{aligned}
U_{\varphi}(\tau+\tau)= & \exp (-i \tau H / \hbar) \exp \left(-i \varphi I^{z}\right) \\
& \times \exp (i \tau H / \hbar) \exp \left(i \varphi I^{z}\right)
\end{aligned}
$$

The signal acquired after a read-out pulse of $\pi / 2, S_{\varphi}(\tau)=$ $\operatorname{Tr}\left[I^{z} U_{\varphi}^{\dagger}(\tau+\tau) I^{z} U_{\varphi}(\tau+\tau)\right]$, is a function of the evolution time $\tau$ and the phase angle $\varphi$. A waiting time $t_{w}$ before the detection ensures the loss of undesired signal in the plane. The observed signal, $S_{\varphi}(\tau)$, is given by

$$
S_{\varphi}(\tau)=\sum_{n} \exp (i \varphi n) S_{n}(\tau)
$$

where $S_{n}(\tau)$ is the multiple quantum coherence order distribution in the Zeeman basis at a given evolution time $\tau$, that can be obtained by Fourier transformation of the acquired signal with respect to $\varphi$. Note that the total signal acquired at $\varphi=0$ is the Loschmidt echo and is equivalent to the addition of all the coherence order intensities at a given time, i.e.,

$$
L E(\tau)=S_{\varphi=0}(\tau)=\sum_{n} S_{n}(\tau) .
$$

A reference signal, or free induction decay (FID), is obtained by the application of a single $\pi / 2$ pulse. The signals $S_{\varphi}(\tau)$, normalized to the FID, satisfy $S_{\varphi}(0)=1$.

The double quantum Hamiltonian $H=H_{\mathrm{DQ}}$ governing the evolution satisfies

$$
H_{\mathrm{DQ}}=-\frac{1}{2} \sum_{i<j} d_{i j}\left(I_{i}^{+} I_{j}^{+}+I_{i}^{-} I_{j}^{-}\right),
$$


where $d_{i j}$ are the dipolar coupling constants:

$$
d_{i j}=\frac{\mu_{0} \gamma^{2} \hbar}{4 \pi r_{i j}^{3}} \frac{\left[1-3 \cos ^{2}\left(\theta_{i j}\right)\right]}{2} .
$$

Here, $\gamma$ is the gyromagnetic ratio, $r_{i j}$ is the spin-spin distance, and $\theta_{i j}$ is the angle between the internuclear vector and the external field $B_{0}$. Experimentally, the Hamiltonian $H_{\mathrm{DQ}}$, is created by using the eight-pulse sequence shown in Fig. 1 [4,9].
In the $\pi / 2$ pulse train, each pulse has duration $t_{p}$ and phases $x$ and $-x$, interspersed with evolution times $\Delta=d-t_{p}$ and $\Delta^{\prime}=2 d-t_{p}$. The full cycle duration is $\tau_{c}=12 d$. In principle, the system evolves by chemical shift $H_{z}$ and secular dipolar interactions $H_{z z}=\sum_{j<k} d_{j k}\left[3 I_{j}^{z} I_{k}^{z}-\vec{I}_{j} \cdot \vec{I}_{k}\right]$. However, during the pulse train, rotations will produce evolutions under $H_{y}$ and $H_{y y}$, combining two Hamiltonians for each time step in the following sequence:

$$
\begin{array}{ccccccccc}
\Delta / 2 & \Delta^{\prime} & \Delta & \Delta^{\prime} & \Delta & \Delta^{\prime} & \Delta & \Delta^{\prime} & \Delta / 2 \\
H_{z} & H_{-y} & H_{-z} & H_{-y} & H_{z} & H_{y} & H_{-z} & H_{y} & H_{z} \\
H_{z z} & H_{y y} & H_{z z} & H_{y y} & H_{z z} & H_{y y} & H_{z z} & H_{y y} & H_{z z}
\end{array} .
$$

As $H_{x x}+H_{y y}+H_{z z}=0$, or equivalently $-H_{x x}=H_{y y}+H_{z z}$, the effective average Hamiltonian obtained after a cycle is

$$
H_{\mathrm{DQ}}=\frac{1}{3}\left(H_{y y}-H_{x x}\right)=-\frac{1}{2} \sum_{i<j} d_{i j}\left(I_{i}^{+} I_{j}^{+}+I_{i}^{-} I_{j}^{-}\right) .
$$

This is a pure double quantum operator in which the zeroquantum flip-flops of the secular Hamiltonian are replaced with the double quantum flip-flips and flop-flops.

It is important to state that this Hamiltonian can be signreversed simply by changing the phases $x$ by $y$ and $-x$ by $-y$ in the pulse train, obtaining $-H_{\mathrm{DQ}}=\frac{1}{3}\left(H_{x x}-H_{y y}\right)$. Note that the nonisotropic dipolar Hamiltonian is the key to obtain the double quantum Hamiltonians and to enable the time-reversal and Loschmidt echo procedures.

\section{EXPERIMENTAL DETAILS}

Adamantane $\left(\mathrm{C}_{10} \mathrm{H}_{16}\right)$ forms a plastic crystal in which the nearly spherical molecules tumble rapidly and isotropically in the solid phase. The motion averages out all the intramolecular dipolar couplings to zero but does not eliminate intermolecular couplings. However, the motion leaves only one distinct coupling between every pair of molecules, reducing the adamantane molecule to a point-dipole source containing 16 spins packed into each point of a face-centered-cubic lattice; i.e., the 16 spins do not interact directly but with other spins in lattice points. This sample involves an infinite network of spin $\frac{1}{2}$ interacting through dipolar interactions [33].

The experiments were performed at $7 \mathrm{~T}$ using a Bruker Avance II spectrometer and a Doty DSI-703 proton dedicated probe with proton background signal reduction. The sample temperature was maintained at $300 \mathrm{~K}$ under static conditions, and the spin lattice relaxation time was determined to be $T_{1}=1.2 \mathrm{~s}$. The $\pi / 2$ pulse was set to $2.2 \mu \mathrm{s}$, the time between evolution and reversion blocks was set to $t_{0}=1 \mu \mathrm{s}$, and the waiting time $t_{w}=1 \mathrm{~ms}$. The excitation and reversion Hamiltonians were generated by repeating $m$ times a block of eight pulses with a fixed duration, $\tau_{\mathrm{DQ}}^{\text {cycle }}$. This cycle time was optimized taking into account the performance of the LE for block durations ranging between 58 and $140 \mu \mathrm{s}$, where the selected optimum block length was $\tau_{\mathrm{DQ}}^{\text {cycle }}=120 \mu \mathrm{s}$.

For excitation times up to $720 \mu \mathrm{s}$, the phase $\varphi$ was incremented in steps $\Delta \varphi=2 \pi / 256$, encoding up to 128 co- herence orders. For excitation times $\tau$ longer than $720 \mu \mathrm{s}$, the increment was $\Delta \varphi=2 \pi / 512$, encoding up to 256 coherence orders.

\section{CLUSTER SIZE DISTRIBUTION MODEL}

To understand the excitation of multiple quantum coherence orders, let us begin with the evolution of $\rho$ in elementary time steps $\delta t$,

$$
\begin{aligned}
\rho(t+\delta t) & =\exp (-i \delta t H / \hbar) \rho(t) \exp (i \delta t H / \hbar) \\
& \cong \rho(t)-i \frac{\delta t}{\hbar}[H, \rho(t)] .
\end{aligned}
$$

This emphasizes the most important features of the evolution. First, that there is a finite propagation time for the interaction $d_{j k}$ between two spins $j$ and $k$. A particular coupling will be able to appreciably influence the dynamics, only when the evolution $d_{j k} \delta t$ is not infinitesimal. Second, the evolution in successive time steps develops a nested hierarchy of commutators. Thus, operators that reflect multispin modes arise simultaneously with the coupling network growth. Examples of a few relevant commutators are

$$
\left[I_{j}^{+} I_{0}^{+}, I_{0}^{z}\right]=-I_{j}^{+} I_{0}^{+}, \quad\left[I_{k}^{-} I_{j}^{-},-I_{j}^{+} I_{0}^{+}\right]=2 I_{k}^{-} I_{j}^{z} I_{0}^{+} .
$$

A further time step is built from nested commutators, for instance,

$$
\begin{aligned}
{\left[I_{l}^{+} I_{j}^{+},\left[I_{k}^{-} I_{j}^{-},-I_{j}^{+} I_{0}^{+}\right]\right] } & =\left[I_{l}^{+} I_{j}^{+}, 2 I_{k}^{-} I_{j}^{z} I_{0}^{+}\right] \\
& =-2 I_{l}^{+} I_{k}^{-} I_{j}^{+} I_{0}^{+} .
\end{aligned}
$$

The product of $K$-spin operators of the form $\cdots I_{m}^{+} I_{l}^{+} I_{k}^{z} I_{j}^{+} I_{0}^{+}$ describe modes in which $K$ spins are interconnected. When the Hamiltonian is a single quantum operator, as $H_{z z}$, the modes of interconnected spins are single quantum operators. Nonsecular Hamiltonians, as $H_{\mathrm{DQ}}$, contain zero and double quantum terms and are able to generate numerous combinations of multiquantum coherences (see Fig. 2).

The most accepted model to extract the number of correlated spins was proposed by Baum et al. [4]. and it relies on assuming that all pathways contributing with an allowed coherence order are weighted with the same probability. Under this assumption, the amplitude of each coherence in the spectrum is related directly to the number of elements of the density matrix with a certain coherence order, given 


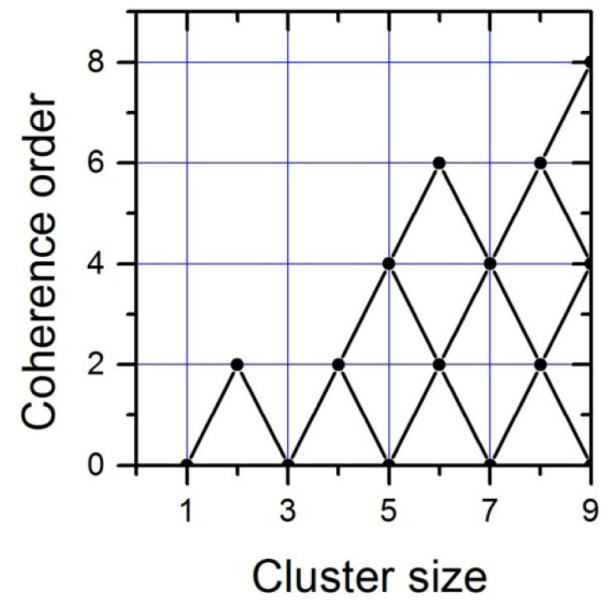

FIG. 2. (Color online) Allowed pathways through the Liouville space for a double quantum evolution.

by a binomial distribution. According to this model, the spectral intensity of an $n$-quantum mode $I(n)$ is given by the combinatorial formula:

$$
\left(\begin{array}{c}
2 K \\
K-n
\end{array}\right)=\frac{(2 K) !}{(K-n) !(K+n) !}
$$

where $K$ is the number of interacting spins and $n$ is the coherence order. For large values of $K$, Stirling's approximation can be used, and the intensity becomes proportional to

$$
I(n, K) \propto e^{-\left(n^{2} / K\right)},
$$

from which the instantaneous effective size of the system, $K$, can be inferred.

The dynamical path that follows the interaction network of spins depends on the Hamiltonian governing that evolution. Figure 2 displays the values of the possible paths given by the selection rules; in the case of the double quantum Hamiltonian $H_{\text {DQ }}[32]$,

$$
\Delta K= \pm 1, \quad \Delta n= \pm 2 .
$$

Note that the evolution develops only even orders of coherence.

In this work, we propose that the coherence orders are described by a distribution function that is assumed as the superposition of Gaussian functions representing clusters of different sizes $K$, with normalized amplitudes $f(K)$ as

$$
S_{n}=\int_{0}^{\infty} d K f(K) e^{-\left(n^{2} / K\right)} .
$$

This distribution can be replaced by a finite sum in the case that the mean sizes are sufficiently different:

$$
S_{n}=\sum_{i=1}^{L} \frac{2 \tilde{A}_{i}}{\sqrt{K_{i} \pi}} e^{-\left(n^{2} / K_{i}\right)} .
$$

With this simplified representation, the coherence order distribution involves the contributions of $L$ clusters having sizes $K_{i}$. The parameters $2 \tilde{A}_{i}$ are the areas of each Gaussian. As the experimental data are normalized to the reference FID, the parameters $\tilde{A}_{i}$ represent the fraction of spins involved in all clusters of size $K_{i}$, relative to the total number of spins detected at time 0 . If $N$ spins contribute to the FID at $\tau=0$, $m_{i}$ clusters of size $K_{i}$ exist for $\tau>0$; then the total number of spins involved in clusters of size $K_{i}$ is $m_{i} K_{i}$. These $m_{i}$ clusters of size $K_{i}$ contribute with a fraction $\tilde{A}_{i}=m_{i} K_{i} / N$ with respect to the total initial spins $N$. The sum of the parameters $\tilde{A}_{i}$ represents the total signal acquired, and is equivalent to the LE,

$$
\sum_{i=1}^{L} \tilde{A}_{i}=S_{\varphi=0}=\mathrm{LE} .
$$

Note that the LE is half of the addition of the Gaussians areas $\left(2 \tilde{A}_{i}\right)$ since only an even order of coherences is generated with the double quantum Hamiltonian. As odd coherence orders have zero intensity, each Gaussian has an area that is the double the one given by the experimental coherence order distribution.

On the other hand, the total number of detected spins $N_{\tau}$ decreases for $\tau>0$ due to decoherence effects and experimental errors. The experimental data normalized to the LE at a given time represent the fraction of spins involved in each cluster size with respect to the total spins detected at that time. Equation (3) can be rewritten in terms of Gaussian functions with areas $2 A_{i}$ as

$$
S_{n}=\sum_{i=1}^{L} \frac{2 A_{i}}{\sqrt{K_{i} \pi}} e^{-\left(n^{2} / K_{i}\right)} .
$$

The new parameters are $A_{i}=m_{i} K_{i} / N_{\tau}$. In this case the sum of the areas is normalized; then $\sum A_{i}=\sum m_{i} K_{i} / N_{\tau}=1$. It is clear that in this simplified model we have considered that the only source of relaxation is the global decoherence described by the LE, whereas different coherence orders could be subject to different multiquantum relaxation mechanisms or even spin diffusion. Nevertheless, numerical calculations of the coherence evolution in small closed systems composed by thermotropic liquid crystals describe very precisely the experimental data when normalization with the LE is carried out [20]. The validity of this assumption in a multispin open system such as adamantane is still an open question; in particular possible errors in the determination of the larger cluster sizes are a subject for further studies. However, as the spin-lattice relaxation time is three orders of magnitude larger than the longest evolution time used, the use of the global LE to account for decoherence is probably the correct choice.

\section{A. Experimental data fitting}

The experimental MQC distributions renormalized to the LE were fitted with Eq. (5), taking $L=2$. Figure 3 shows the experimental points for the MQC distribution at $960 \mu \mathrm{s}$ together with the fitting functions of Eq. (5), where the inset shows the global fitting. It represents a good agreement with the experimental data for all the coherence orders except for $n=0$, the zero quantum coherence order (ZQC), which was excluded in the fittings at all the evolution times. Nevertheless, we compute a zero-order difference (ZOD) as the distance between the experimental value $\left(S_{n=0}\right)$ and the value given by the fitting curve at $n=0$, as it could provide useful information on decoherence, see inset in Fig. 3.

Figure 4 shows the experimental MQC distributions for $\tau=960,1080,1200,1320 \mu$ s together with their corresponding fittings using Eq. (5) with two-Gaussian functions 


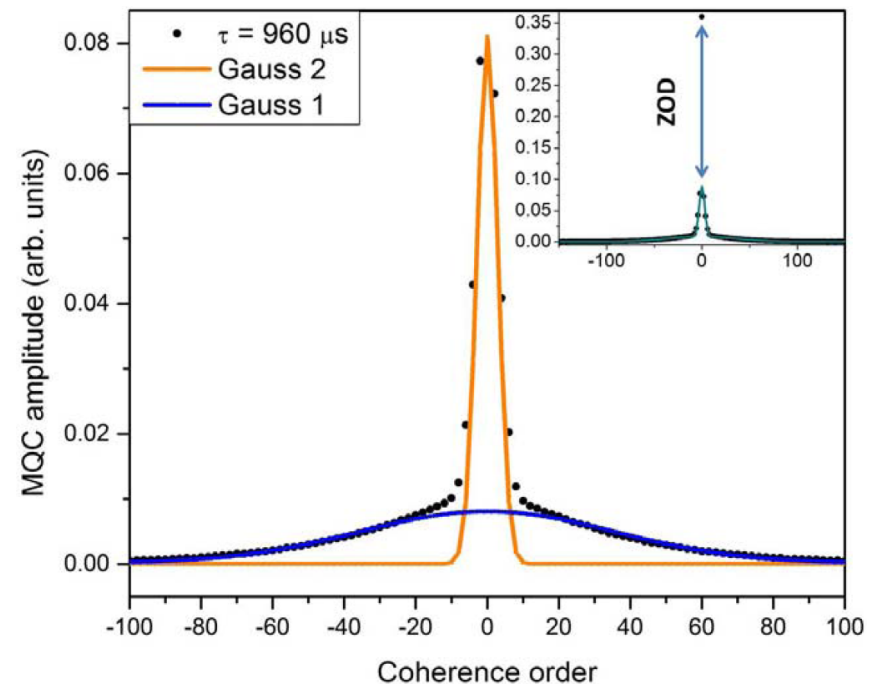

FIG. 3. (Color online) Experimental MQC distribution $S_{n}$ at $960 \mu \mathrm{s}$, together with the fitting functions for $L=2$. Gauss 1 and Gauss 2 have been displayed separately. The inset displays the total fitting to the experimental curve, i.e., the sum of the two-Gaussian functions. The arrow in the inset marks the zero-order difference (ZOD).

$(L=2)$. In previous works in adamantane, MQC distributions have been represented by a single function $(L=1)$, using a Gaussian or an exponential function [9,20,24,34]. It is evident that a single function cannot fit the behavior of the experimental MQC for all the orders (see Appendix). Figure 4 shows that for times longer than $1 \mathrm{~ms}$, the intensity corresponding to the higher coherence orders $(n \approx 120)$ drastically decreases falling into the noise level. Thus, the associated cluster size $K_{1}$ (Gauss 1 in Fig. 3) is a parameter that has an increasing relative error in its determination.

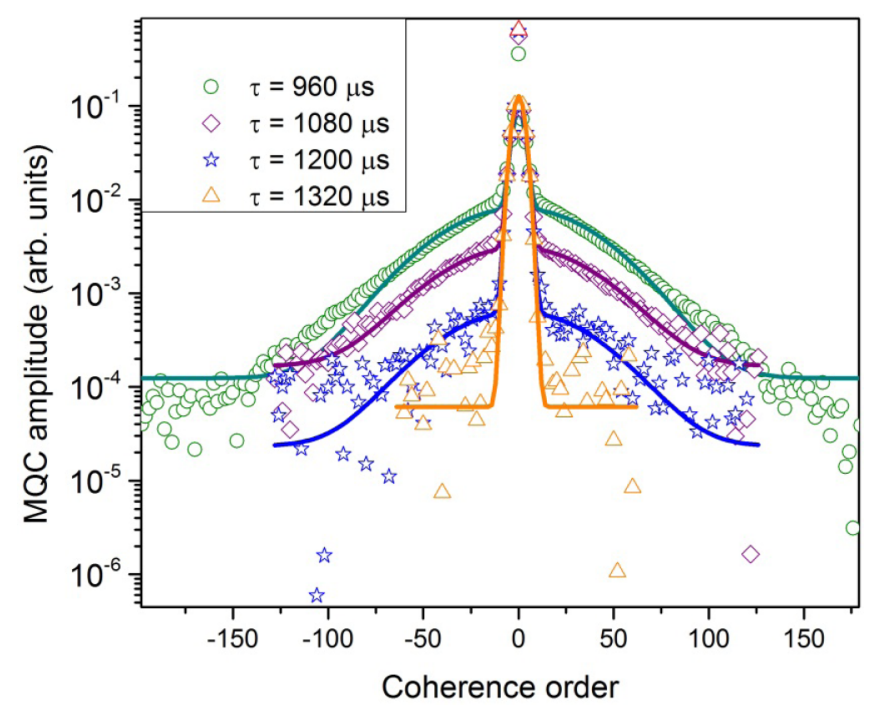

FIG. 4. (Color online) Experimental MQC distributions at different evolution times (symbols) together with the corresponding total fittings to Eq. (5) (solid lines) in logarithmic scale. In all the cases, two-Gaussian functions $(L=2)$ have been used. In all cases the coefficient of determination, $R^{2}$, is better than $99 \%$.

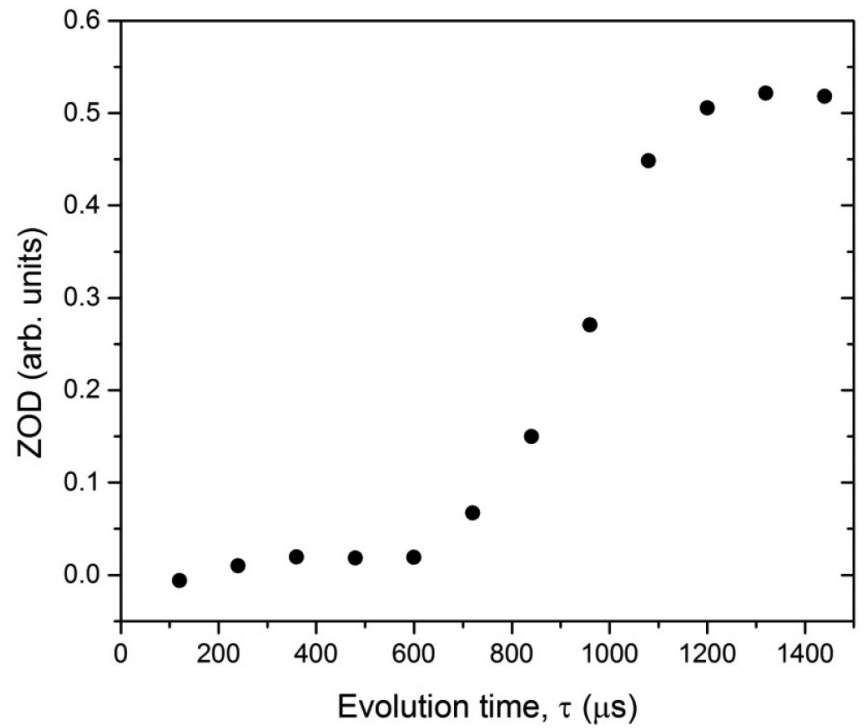

FIG. 5. ZOD as a function of evolution time.

Figure 5 displays the behavior of the ZOD as a function of the evolution time. It can be observed that ZOD increases with $\tau$ up to $1.2 \mathrm{~ms}$ where a constant value is reached. This fact can be interpreted taking into account that when the evolution time increases, a bigger fraction of spins lose their encoding phase, but still produce an additional contribution to the experimental ZQC from the $z$ magnetization.

\section{RESULTS AND DISCUSSION}

By using the model proposed in Eq. (5) for the twoGaussian fittings $(L=2)$ relevant information about the multispin system evolution can be extracted. Figure 6 displays the evolution of the number of correlated spins $K_{i}$ in each group of clusters. Two different behaviors can be observed. On one side $K_{1}$, associated to large clusters, has a nearly exponential growth, reaching more than 2500 spins at around

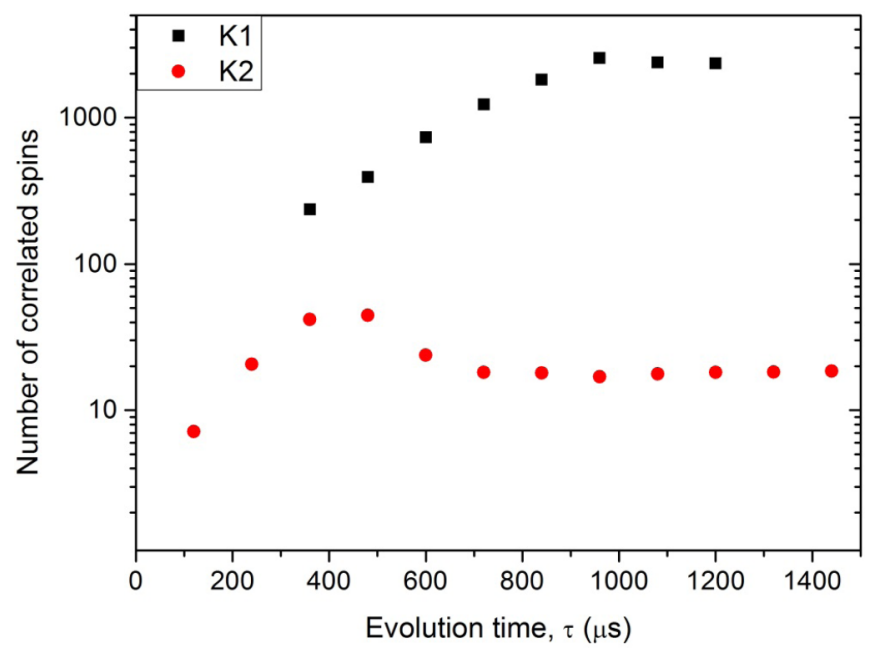

FIG. 6. (Color online) Number of correlated spins (cluster size) for clusters $i=1,2$ as a function of time, representing the distribution of correlations in the system. 


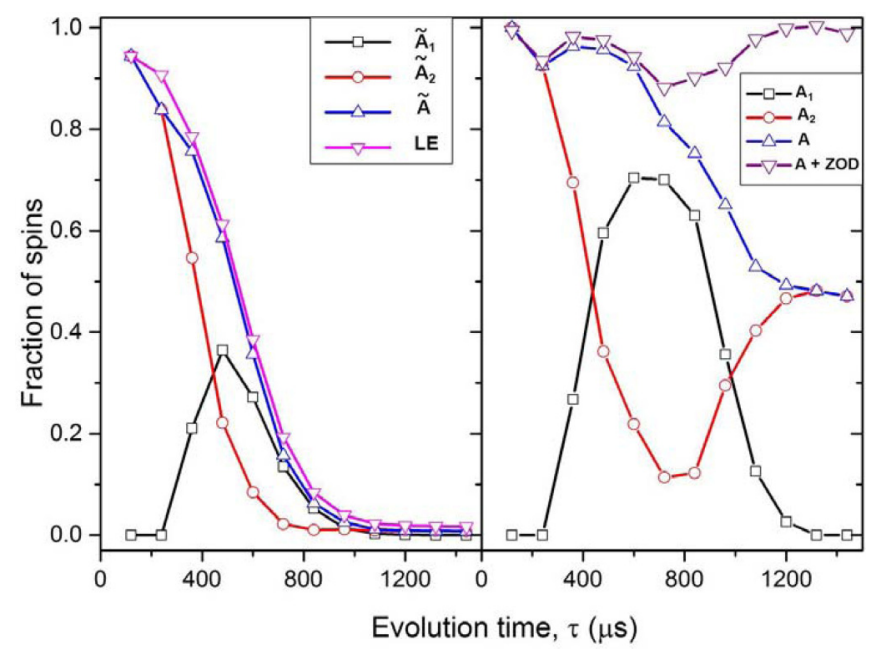

FIG. 7. (Color online) (Left) parameters $\tilde{A}_{i}$ for $i=1,2$, together with $\tilde{A}=\tilde{A}_{1}+\tilde{A}_{2}$ and the LE as a function of the evolution time. (Right) parameters normalized to the LE, $A_{i}=\tilde{A}_{i} / L E$ for $i=1,2$.

$1 \mathrm{~ms}$. The behavior of these big clusters corresponds to the already reported observations of Refs. [9,20,24,34], where the MQC distribution was fitted to a single function (e.g., a Gaussian, $L=1$ ) using only high orders of coherence. The behavior of $K_{1}$ shows an apparent saturation for $\tau$ longer than $1 \mathrm{~ms}$. This fact can be associated to an almost disappearance of the signal under the noise background for the higher orders as observed in Fig. 4, precluding any further analysis from the noisy tails.

For the small clusters, represented by $i=2$, a remarkable behavior is revealed. The cluster size $K_{2}$ displays an initial increase in the first $400 \mu$ s followed by stabilization at longer times, with $K_{2} \approx 18$. Note that these clusters have not been considered in previous works, where the lower orders of coherence were excluded from the fittings, as, for instance, in Ref. [34]. For times close to $400 \mu$ s it is difficult to separate the information from each cluster, as both clusters have similar sizes. This fact could be the source of the oscillation observed in $K_{2}$.

We now turn our attention to the contribution of each cluster to the overall signal. Figure 7 (left) displays the parameters $\tilde{A}_{i}$ for $i=1,2$ as a function of the evolution time, together with the total spins determined from the fittings $\left(\tilde{A}=\tilde{A}_{1}+\tilde{A}_{2}\right)$ and the LE. On the right panel, parameters $A_{i}$, together with $A=A_{1}+A_{2}$, are displayed. As previously mentioned, these parameters represent the fraction of spins that are detected and that participate in clusters of each size. It can be observed that $\tilde{A}$ follows the trend of the LE curve as a function of time; the difference between them can be accounted for by inspection of the evolution of the renormalized data. It is clear that in an ideal experiment, free of decoherence and pulse imperfections, the data renormalized to the LE should have a constant value $A=1$. This is the case at short times (up to $\sim 500 \mu \mathrm{s}$ ); however, for longer times $A$ decreases down to 0.5 due to the ZOD (Fig. 5), which contributes to the LE but not to the fittings. In this way, $A+\mathrm{ZOD} \approx 1$ for all times, within a $10 \%$ margin of error. It must be noted that ZOD becomes relevant after $720 \mu \mathrm{s}$; at this evolution time the contribution of the larger clusters $\left(A_{1}\right)$ starts to decrease and the contribution of the smaller clusters becomes relatively larger. This is a clear indication that decoherence is more important for the bigger clusters of correlated spins, i.e., for the more entangled states. For increasing evolution times, ZOD represents almost $50 \%$ of the total signal. It is important to note that the remaining $50 \%$ is the relevant information encoded in the MQC distribution; however, this information is stored mainly in the small clusters $\left(K_{2}\right)$. The intensity of the large clusters in MQC completely falls under the noise level and cannot be determined for these evolution times. Note that at this stage the LE is below $5 \%$ of the initial signal intensity (Fig. 7, left).

By comparing Figs. 6 and 7 a final insight into the system dynamics can be obtained. At short times all the spins contribute to small clusters; as the evolution time increases large clusters appear, reducing the percentage of spins contributing to small clusters. For instance, at $\tau=360 \mu \mathrm{s}, 70 \%$ of the detected spins belong to clusters of nearly 40 spins. On the other hand, $30 \%$ of the total signal belongs to clusters of 230 correlated spins. For evolution times up to around $800 \mu \mathrm{s}$ the information stored in the density matrix is exchanging the spins involved in the small clusters toward the large ones. After $800 \mu$ s there is an apparent reversion of this behavior. The increase in the relative contribution of the small clusters (Fig. 7, right) must not be misunderstood as a reversal in the system dynamics, but is attributed to the fact that large clusters lose coherence more easily, while the information in the small clusters can be recovered by renormalization with the LE. A global loss of information due to decoherence of the big clusters is evidenced by the abrupt fall of the LE, as shown in Fig. 7 (left).

For times longer than $1.2 \mathrm{~ms}$, the information from the large clusters is completely lost. In Fig. 4 it is possible to see how the intensity of the coherence order distribution for clusters larger than 2000 spins (1.32 ms) is below the noise level. For these times the coherence order distribution function is only provided by a small fraction of the total initially detected spins, which have maintained their coherence, contributing to small clusters of around 18 spins. Naturally, these small clusters are also subject to decoherence and their decay follows the LE. While the true nature of these small clusters still remains to be determined, a possible hypothesis is that they are constituted by near neighbors in the adamantane lattice (12 molecules) plus the next-nearest neighbors (six molecules). We rather favor an alternative hypothesis that, although the interactions between protons within a molecule (16 spins) are averaged by rapid rotations, they could become correlated via interactions with the protons in the next neighbors, in a kind of second-order interaction.

\section{CONCLUSIONS}

In this work we proposed an improved approach for the analysis of multiple quantum coherence orders distributions, assuming that the evolving multispin system can be considered as an ensemble of clusters of correlated spins with different sizes. Each cluster was characterized by two parameters: the cluster size, which is determined by spin counting, and the fraction of spins belonging to the cluster, which was determined by normalizing with the LE. Each cluster develops at a rate characteristic of the Hamiltonian governing the evolution, and the selection rules imposed by this Hamiltonian. 
Furthermore, the behavior of the fractions of clusters provided an insight into how the correlations involving different quantum coherence orders are affected by decoherence. That is, when decoherent interactions perturb the state of a cluster there is a loss of information on all the coherence orders involved, which are determined by the number of correlated spins $K$. Then, lower coherence orders are associated to all cluster sizes in the sample, while higher orders belong only to larger clusters. Consequently, higher coherence orders are far more sensitive to decoherent interactions than the lower ones, which is consistent with the observation that decoherence affects the coherence orders in a different way. Assessing these issues in greater detail opens an alternative field for experimental and theoretical study.

\section{ACKNOWLEDGMENTS}

We acknowledge the financial support from Consejo Nacional de Investigaciones Científicas y Técnicas (CONICET), Secretaría de Ciencia y Técnica de la Universidad Nacional de Córdoba (SECyT-UNC), and Agencia Nacional de Promoción Científica y Tecnológica (ANPCyT).

\section{APPENDIX}

The results for a fitting of a MCQ distribution with a single Gaussian function compared to the results with two Gaussian functions are shown in Fig. 8 for an evolution time of $1080 \mu \mathrm{s}$. As the contribution of the two cluster types differs in several orders of magnitude in intensity, fitting with a single Gaussian function will describe only the smaller clusters. Figure 8 (right) shows that a two-Gaussian

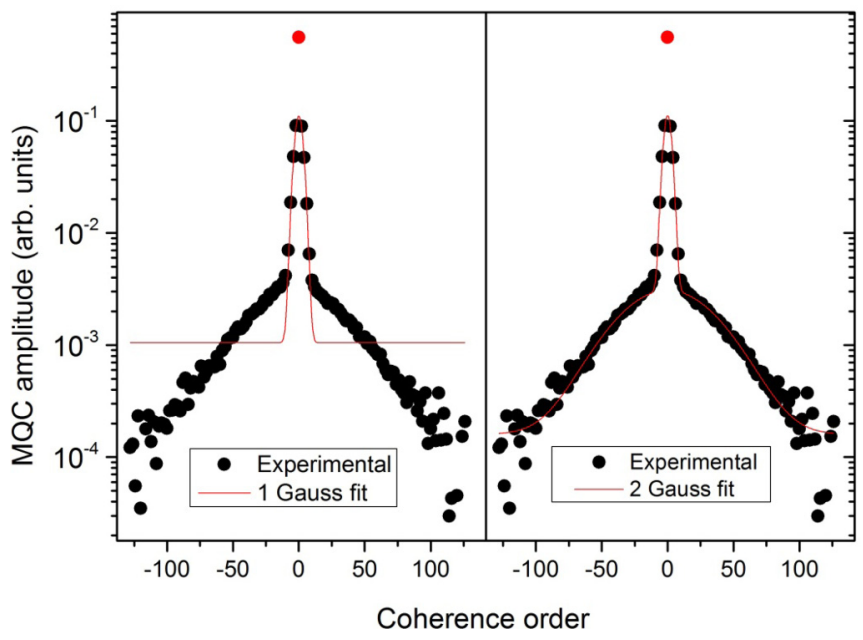

FIG. 8. (Color online) Experimental data for $\tau=1080 \mu \mathrm{s}$. (Left) Fitting with a single-Gaussian function: $R^{2}=0.9937$. (Right) Fitting with two-Gaussian functions: $R^{2}=0.9993$.

function fitting describes the distribution of coherences more accurately. Slight fitting improvements can be achieved by performing a multi-Gaussian function. In this case, the larger cluster decomposes in various components maintaining the exponential growth in the number of correlated spins. Remarkably, the saturating small cluster contribution remains robust against these changes. This reinforces the physical nature of the small clustering, as associated to a natural hierarchy in the interactions network.
[1] R. M. Serra and I. S. Oliveira, Philos. Trans. R. Soc., A 370, 4615 (2012).

[2] R. R. G. Ernst, G. Bodenhausen, and A. Wokaun, Principles of Nuclear Magnetic Resonance in One and Two Dimensions, International Series of Monographs on Chemistry (Clarendon Press, Oxford, 1987).

[3] H. Cho, T. D. Ladd, J. Baugh, D. G. Cory, and C. Ramanathan, Phys. Rev. B 72, 054427 (2005).

[4] J. Baum, M. Munowitz, A. N. Garroway, and A. Pines, J. Chem. Phys. 83, 2015 (1985).

[5] W.-K. Rhim, A. Pines, and J. S. Waugh, Phys. Rev. Lett. 25, 218 (1970).

[6] C. A. Michal and R. Tycko, J. Chem. Phys. 114, 409 (2001).

[7] C. M. Sánchez, H. M. Pastawski, and P. R. Levstein, Phys. B: Condens. Matter 398, 472 (2007).

[8] P. R. Levstein, G. Usaj, and H. M. Pastawski, J. Chem. Phys. 108, 2718 (1998).

[9] H. G. Krojanski and D. Suter, Phys. Rev. Lett. 93, 090501 (2004).

[10] H. G. Krojanski and D. Suter, Phys. Rev. Lett. 97, 150503 (2006).

[11] W.-K. Rhim, A. Pines, and J. S. Waugh, Phys. Rev. B 3, 684 (1971).

[12] S. Zhang, B. H. Meier, and R. R. Ernst, Phys. Rev. Lett. 69, 2149 (1992).

[13] W. H. Zurek, Rev. Mod. Phys. 75, 715 (2003).
[14] A. A. Goussev, R. Jalabert, H. M. Pastawski, and D. A. Wisniacki, Scholarpedia 7, 11687 (2012).

[15] G. Usaj, H. M. Pastawski, and P. R. Levstein, Mol. Phys. 95, 1229 (1998).

[16] H. M. Pastawski, P. R. Levstein, G. Usaj, J. Raya, and J. Hirschinger, Phys. A (Amsterdam, Neth.) 283, 166 (2000).

[17] P. R. Zangara, A. D. Dente, P. R. Levstein, and H. M. Pastawski, Phys. Rev. A 86, 012322 (2012).

[18] R. A. Jalabert and H. M. Pastawski, Phys. Rev. Lett. 86, 2490 (2001).

[19] P. R. Levstein, A. K. Chattah, H. M. Pastawski, J. Raya, and J. Hirschinger, J. Chem. Phys. 121, 7313 (2004).

[20] C. M. Sánchez, P. R. Levstein, R. H. Acosta, and A. K. Chattah, Phys. Rev. A 80, 012328 (2009).

[21] E. Rufeil-Fiori, C. M. Sánchez, F. Y. Oliva, H. M. Pastawski, and P. R. Levstein, Phys. Rev. A 79, 032324 (2009).

[22] C. Ramanathan, H. Cho, P. Cappellaro, G. S. Boutis, and D. G. Cory, Chem. Phys. Lett. 369, 311 (2003).

[23] S. W. Morgan, V. Oganesyan, and G. S. Boutis, Phys. Rev. B 86, 214410 (2012).

[24] J. Baum and A. Pines, J. Am. Chem. Soc. 108, 7447 (1986).

[25] H. Cho, D. G. Cory, and C. Ramanathan, J. Chem. Phys. 118, 3686 (2003).

[26] W. Zhang, P. Cappellaro, N. Antler, B. Pepper, D. G. Cory, V. V. Dobrovitski, C. Ramanathan, and L. Viola, Phys. Rev. A 80, 052323 (2009). 
[27] C. M. Sánchez, Ph.D. thesis, Universidad Nacional de Córdoba, Argentina, 2011.

[28] G. Kaur, A. Ajoy, and P. Cappellaro, New J. Phys. 15, 093035 (2013).

[29] P. J. Hore, J. A. Jones, and S. Wimperis, NMR, the Toolkit (Oxford University Press, Oxford, 2000).

[30] F. Galton, Natural Inheritance (MacMillan, London, 1889).
[31] G. A. Álvarez and D. Suter, Phys. Rev. Lett. 104, 230403 (2010).

[32] M. Munowitz and A. Pines, in Advances in Chemical Physics, edited by I. Prigogine and S. A. Rice (John Wiley \& Sons, Inc., Hoboken, NJ, 1986), pp. 1-152.

[33] I. Schnell and H. W. Spiess, J. Magn. Reson. 151, 153 (2001).

[34] G. A. Álvarez and D. Suter, Phys. Rev. A 84, 012320 (2011). 